\title{
ROBUST SHAPE CONTROL IN A SENDZIMIR COLD-ROLLING STEEL MILL
}

\author{
D.G. Bates ${ }^{\star}$, J.V. Ringwood ${ }^{\star \star}$ and A.M. Holohan ${ }^{\star \star *}$ \\ *Department of Engineering, University of Leicester, Leicester LEI 7RH, UK \\ **School of Electronic Engineering, Dublin City University, Glasnevin, Dublin 9, Ireland (ringwood@eeng.dcu.ie) \\ ${ }^{* * *}$ Department of Mathematics, University of Twente, 7522NP Enschede, The Netherlands
}

(Received February 1996; in final form October 1997)

\begin{abstract}
The shape control problem for a Sendzimir 20-roll cold rolling steel mill is characterised by operation over a wide range of conditions arising from roll changes, changes in rolling schedules and changes in material gauge, width and hardness. Previous approaches to the problem suggest storing a large number of precompensator matrices to cater for the full range of operating conditions. This paper, on the other hand, attempts to synthesise a controller which is optimally robust to changes in the conditions associated with the rolling cluster, resulting in a reduced storage requirement for the controlling computer. The performance of the robust controller is evaluated via nonlinear simulation.
\end{abstract}

Copyright $\odot 1997$ Elsevier Science Ltd

Keywords. Steel manufacture, shape control, robust control, gain scheduling

\section{INTRODUCTION}

Accurate control of the shape (internal stress distribution) of steel strip in cold rolling presents a significant challenge, due to the multi-pass, multischedule nature of the activity. The different passes and schedules (approx. 2500 in all) required to achieve a given final gauge for different grades and widths of rolled strip involve variations in mill setup, such as roll diameters and strip speed, and changes in material characteristics, such as input and output gauges for each pass, strip width and material hardness. These cause significant (up to 300\%) changes in the mill model parameters, which point clearly to a requirement for a number of controllers.

The Sendzimir mill (see Fig. 1) is a reversing mill, and a separate schedule containing a number of passes is specified for each different material rolled. A schedule can contain from 4 to 15 passes through the rolling cluster. Each pass involves different entry and exit gauges, with minor changes in the material hardness from pass to pass.

To date, the approach has been to design controllers using traditional multivariable techniques for a set of nominal cases (e.g., every schedule), and then employ a test to check for controller stability for schedules and passes outside this nominal set (Ringwood, et al., 1990; Ringwood, 1995).

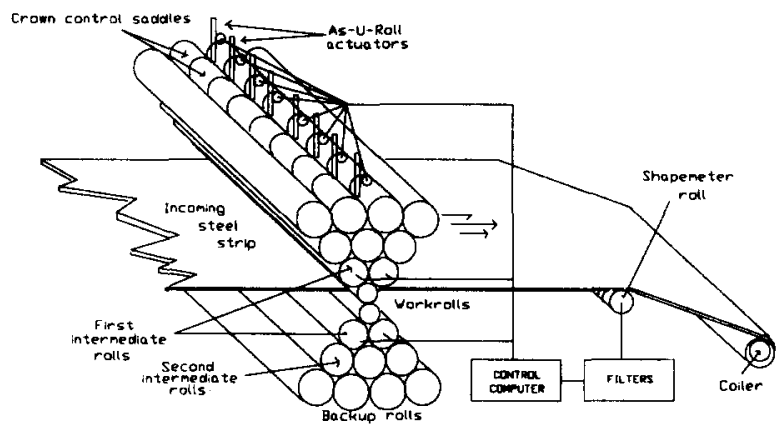

Fig. 1. 20-roll cold rolling Sendzimir mill

However, the deficiencies of this approach are (a) little attempt is made to actively build in robustness to model variations, resulting in possible wide variations in performance (although stability may be retained), and (b) no systematic method for scheduling different controllers across different passes and schedules is obvious. Current industrial practice is to employ some decoupling and feedforward techniques with PID controllers (Jaeckel, 1993). 
This paper attempts to actively build in robustness to model parameter variations, due to pass and schedule changes, and clearly identify a scheduling strategy which can be implemented. In addition, other sources of model imperfection are addressed. The approach relies on a problem formulation in $\mathrm{H}_{\infty}$, where an attempt is made to guarantee robust stability over an unstructured model uncertainty due to pass changes, while maintaining reasonable performance (small sensitivity function) and high-frequency measurement noise rejection.

\section{SENDZIMIR MILL MODEL}

The Z-mill has an ASEA 'Stressometer' for measuring the differential tension (or stress) profile across the strip. This device is mounted $2.91 \mathrm{~m}$ downstream of the roll gap, and produces 8 (modelled) output measurements. Four pressure measurements per revolution of this device are provided, causing a fourperiod-per-revolution sinusoid to be superimposed on the output signal $(40 \mathrm{~Hz}$ at a speed of $10 \mathrm{~m} / \mathrm{sec}$.). Further noise on the output signal is introduced, due to the $2 \mathrm{kHz}$ magnetising currents used with the pressure sensors.

Shape actuation is effected via the 'As-U-Rolls' (AURs), which provide the equivalent of 8 independent (but equally spaced) point loads. This generates roll bending, causing differential elongation of the strip, thus influencing the shape profile.

The Z-mill model, therefore, has 8 outputs and 8 inputs. The rolling cluster is the most complex part of the system and accounts for all of the interaction between the 8 (modelled) paths in the system. A linearized gain matrix $\left(\mathrm{G}_{\mathrm{a}}\right)$ relates changes in the rollgap shape profile to changes in the positions of the AURs (Gunawardene, 1982). An independent study by Dutton (1983) has confirmed the structure of the mill matrices produced by Gunawardene's model, but the models differ in the absolute values of the determined gains. One focus of the controller developed here will be to provide robustness to the uncertainty in these d.c. gains.

Diagonal dynamic blocks account for the actuators, strip dynamics (between roll-gap and shapemeter) and the shapemeter filters. The mill model is therefore of the form:

$$
y=p(s) G_{a} f_{a}\left(u_{a}\right), G_{a} \varepsilon \Re^{8 \times 8}
$$

where $p(s)$ includes dynamics due to the strip and shapemeter, and the nonlinear function $\mathrm{f}_{\mathrm{a}}($.) represents the AUR actuators (see Fig. 2). An actuator linearisation technique (Ringwood, 1994) is applied to the nonlinear actuators, resulting in a first-order linear response for each actuator with a time constant of 2 secs.

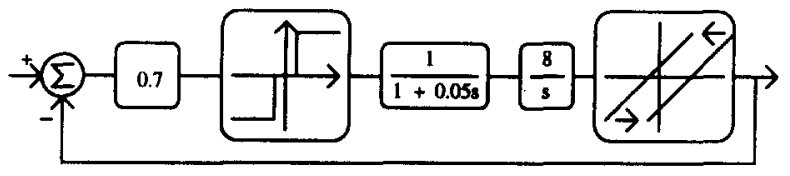

Fig. 2. As-U-Roll actuator block diagram

The resulting overall mill dynamics are therefore given by:

$$
g(s)=\frac{e^{-0.582 s}}{(1+1.064 s)(1+0.74 s)(1+2 s)}
$$

for a medium strip speed $(\approx 10 \mathrm{~m} / \mathrm{s})$. A disturbance, $\mathrm{d}(\mathrm{s})$, is included in the mill model to account for the shape of the incoming steel strip.

The scalar dynamic transfer function, $p(s)$, varies with strip speed, while the mill matrix, $G_{a}$, varies with mill setup (and therefore with pass and schedule no.). In this paper, an attempt will be made to design a controller which can cover all the (six) passes of a given schedule. The controller design is based on the nominal (average) $G_{a}$ of:

$G_{.}=\left[\begin{array}{cccccccc}4.3907 & 4.9866 & -0.07199 & -2.1837 & -2.3299 & -2.0186 & -1.7916 & -1.7943 \\ 0.6112 & 25487 & 2.5138 & 0.2207 & -15248 & -1.9372 & -1.7115 & -1.7027 \\ -0.7673 & 0.4553 & 2.7242 & 1.7667 & -05024 & -1.7515 & -1.7883 & -1.7682 \\ -1.0494 & -1.0781 & 1.1593 & 2.6865 & 1.5551 & -0.6776 & -1.7538 & -1.7282 \\ -0.9135 & -1.6900 & -0.7009 & 1.4843 & 2.7079 & 1.2133 & -1.1479 & -1.1449 \\ -0.7882 & -1.7710 & -1.6810 & -0.3389 & 1.9747 & 2.7206 & 0.4609 & 0.4541 \\ -0.7566 & -1.7308 & -1.9495 & -1.5653 & 0.0505 & 2.3465 & 2.6623 & 2.6831 \\ -0.8345 & -1.8083 & -1.9580 & -2.2416 & -1.9845 & -0.1392 & 4.9882 & 4.9173\end{array}\right]$

Mill matrices for passes 1 and 6 of the schedule are given in the Appendix.

\section{ROBUST CONTROLLER DESIGN}

\subsection{Design Framework}

Controller design in the $\mathrm{H}_{\infty}$ framework provides a guarantee of stability within a given set of model perturbations arising from pass or schedule changes (Maciejowski, 1989). Further objectives include the achievement of good dynamic performance across the set of perturbed plants, and the attenuation of measurement noise and disturbances. Tradeoffs and conflicts arising from these different requirements are resolved using the weighting functions $W_{1}(s)$ and $\mathrm{W}_{2}(\mathrm{~s})$ in the $\mathrm{H}_{\infty}$ cost function:

$$
J=\left\|\begin{array}{l}
W_{1} S(s) \\
W_{2} T(s)
\end{array}\right\|
$$

where:

$$
S(s)=(1+G K(s))^{-1}
$$

is the system sensitivity function, which determines the disturbance-rejection properties of the system, and 


$$
T(s)=G K(s)(1+G K(s))^{-1}
$$

is the complementary sensitivity function, which determines robust stability and measurement (shapemeter) noise attenuation. The components considered in a robust control design are detailed in Fig. 3. A further issue in weight selection is the requirement that the closed-loop bandwidth rolls off in frequency before the phase effects of the pure delay term in eq. (2) become significant. This is achieved using $\mathrm{W}_{2}(\mathrm{~s})$.

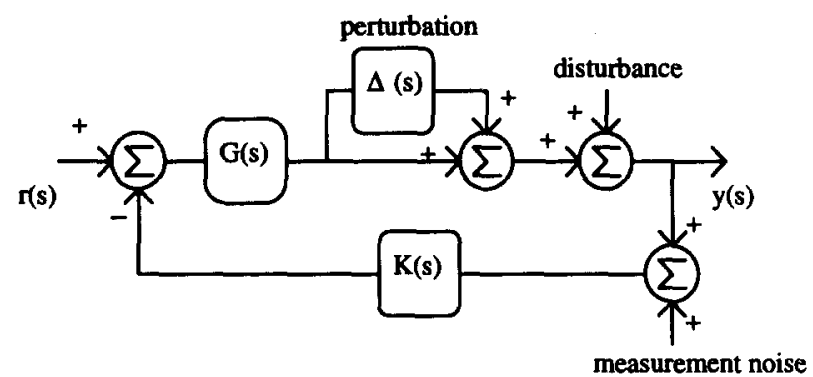

Fig. 3. Plant configuration for robust control design

Robust stability is guaranteed by ensuring that the weight $\mathrm{W}_{2}(\mathrm{~s})$ overbounds the plant (multiplicative) perturbation in the maximum singular value sense as:

$$
\bar{\sigma}\left[W_{2}(j \omega)\right] \geq \bar{\sigma}[\Delta(j \omega)] \quad \forall \quad \omega \geq 0
$$

where

$$
G(s)=G_{\text {nom }}(s)(I+\Delta(s)) .
$$

\subsection{Reduction of Plant Dimension}

Examination of the mill matrix, $G_{a}$, indicates singularity problems due to a significant spread in its singular values. In addition, an order of magnitude difference exists between the four largest and four smallest singular values. As an example, the singular value spectrum of the nominal plant mill matrix $G_{a}$ is:

\{12.3568 $9.11204 .91251 .56250 .3306 \quad 0.21010 .02590 .0051\}$.

This poses a significant problem for an $\mathrm{H}_{\infty}$ design, since the sensitivity function, $S(s)$, will be always close to unity in the directions of the small singular values (i.e., the product $\mathrm{GH}(\mathrm{s})$ is approximately zero in those directions). This suggests a reparameterisation of the plant in terms of the four most significant singular values. Partition the plant as:

where:

$$
G(s)=g(s)\left[U_{1} U_{2}\right]\left[\begin{array}{cc}
\Sigma_{1} & 0 \\
0 & \Sigma_{2}
\end{array}\right]\left[\begin{array}{l}
V_{1}^{T} \\
V_{2}^{T}
\end{array}\right]
$$

$$
U_{1}, U_{2}, V_{1}, V_{2} \in \Re^{8 \times 4} \quad, \quad \Sigma_{1}, \Sigma_{2} \in \Re^{4 \times 4} .
$$

A parameterisation $\mathrm{U}_{1}{ }^{\mathbf{T}}$ is now applied to the mill output shape profile, while the control input is parameterised using $V_{1}$. The $H_{\infty}$ design is now concentrated on the reduced-dimension system, given by:

$$
G_{r e d}(s)=g(s) U_{1}^{T} G_{a} V_{1}
$$

Such a parameterisation is consistent with previous approaches (Ringwood, 1995), motivated by rolling practice considerations.

\subsection{Controller Design}

The $\mathrm{H}_{\infty}$ controller design was now performed using the reduced plant in (9) against the objectives and considerations set out in Section 3.1. The weighting functions are intuitively chosen as:

$$
W_{1}(s)=\frac{10\left(10^{-5} s+1\right)}{10^{2} s+1} I_{4}, W_{2}(s)=\frac{0.2774\left(10^{-3} s+1\right)}{10^{-6} s+1} I_{4}
$$

and are shown together with $\overline{\sigma(\Delta(j \omega))}$ in Fig. 3.

$W_{1}(s)$ is chosen to:

- Penalise sensitivity, $\mathrm{S}(\mathrm{s})$, at low frequency, giving good d.c. disturbance rejection (step changes in incoming strip shape profile due to welds), and

- Ensure that system performance (dynamic response) is maintained in spite of parameter variations at low frequency due to (a) variations in $G_{2}$ due to schedule and pass changes and (b) modelling errors in $G_{a}$.

$\mathrm{W}_{2}(\mathrm{~s})$ is chosen to:

- Ensure robust stability by covering $\Delta(s)$ i.e. that condition (6) is met, and

- Attenuate high-frequency (shapemeter) measurement noise, by driving $\mathrm{T}(\mathrm{s})$ down at high frequency.

In addition, the relative positions of $W_{1}(s)$ and $W_{2}(s)$ determine the closed-loop bandwidth, controlling the dynamic response to setpoints and disturbances.

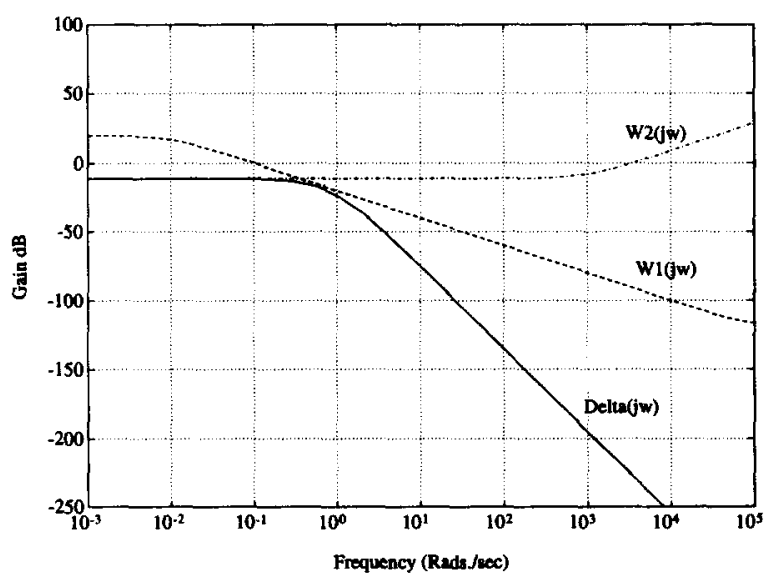

Fig. 4. Weighting functions and perturbation. 
The software used in the controller optimisation was based on the MATLAB® Robust Control Toolbox (Safonov and Chiang, 1988), with the derived controller detailed in the Appendix. A block diagram of the closed-loop system is shown in Fig. 5. Note that the shape-control problem is basically a regulator problem, since the desired shape (stress) profile in the output strip is uniform, i.e. zero at all points.

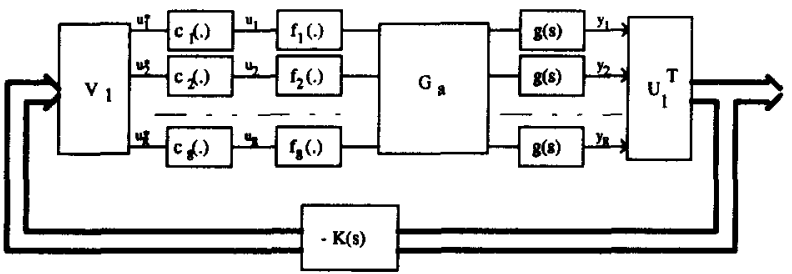

Fig. 5. Closed-loop system

\section{CONTROLLER PERFORMANCE ANALYSIS}

Nominal performance and the achievement of specifications were checked using frequency-response plots, as given in Section 4.1. However, to assess the added effects of neglected time delay and residual nonlinearity in the actuators, nonlinear simulation tests were used to demonstrate realistic controller performance.

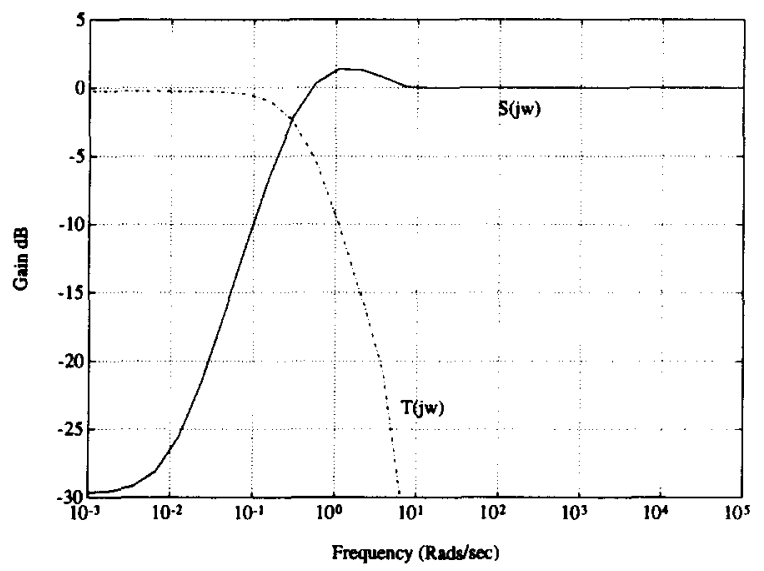

Fig. 6. Sensitivity and complementary sensitivity functions

\subsection{Achievement of design objectives}

Figure 6 shows the system sensitivity function, $S(j \omega)$, and the complementary sensitivity function, $T(j \omega)$. Note that $S$ drops to $-30 \mathrm{~dB}$ at low frequency, ensuring good d.c. disturbance rejection and insensitivity to d.c. plant-parameter variations. The profile of the complementary sensitivity function, which also specifies the closed-loop transfer function, has a $3 \mathrm{~dB}$ bandwidth of approx. $0.1 \mathrm{rads} / \mathrm{sec}$, giving a reasonable closed-loop bandwidth, while providing good attenuation of high-frequency shapemeter noise. In addition, the closed-loop transfer function rolls off before the phase effects of the time delay in eq. (2) become significant.
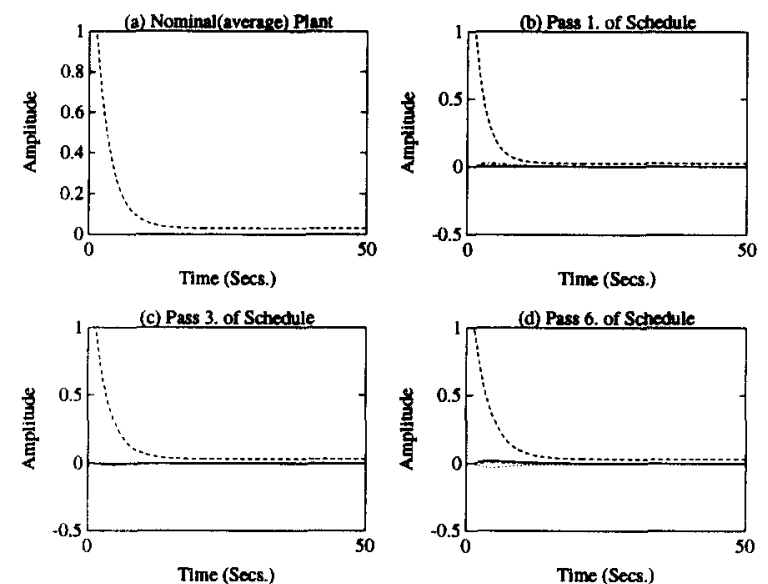

Fig. 7. Disturbance-rejection properties of system

To indicate the transient response and robustness of the system, a step disturbance in path 2 of the idealised (linear, delay-free) system was introduced, and the output reponse for each path is shown in Fig. 7 (a). Robustness to variations in $G_{a}$ is demonstrated by observing the response for 3 different $G_{a}$ matrices, corresponding to passes 1 (Fig. 7 (b)), 3 (Fig. 7 (c)) and 6 (Fig. 7 (d)) of a 6-pass schedule. It is seen that nominal performance and disturbance decoupling are preserved to a good degree of accuracy in all cases.

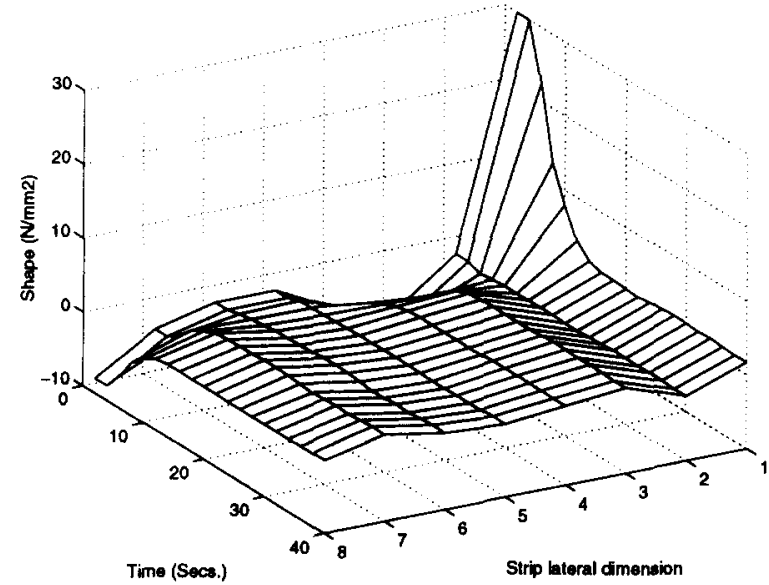

Fig. 8. Shape control for nominal system

\subsection{Simulation results}

The controller developed above was now simulated with a simulation model of the mill, containing the transport delay (which was ignored in the design), nonlinear actuators together with their linearising precompensators, and a realistic incoming strip shape disturbance. The shape profile variations are shown in Fig. 8 for the nominal system, demonstrating the relatively high-order residual shape profile. The desired target profile is flat, translating into the requirement that the strip be free from internal stress. 
Note that, by virtue of the parameterisation in eq. (9), no attempt is made to control the high-order profiles, which accords with normal rolling practice. Parametric shape variations (with desired values of zero), which are shown as the output in Fig. 5, are given in Fig. 9. These plots confirm the results obtained in the previous section, while Fig. 10, which shows the parametric shape profile variations for the nominal controller used in conjunction with $G_{a}$ for Pass 6, verifies the retention of robust stability and performance which is very close to nominal.

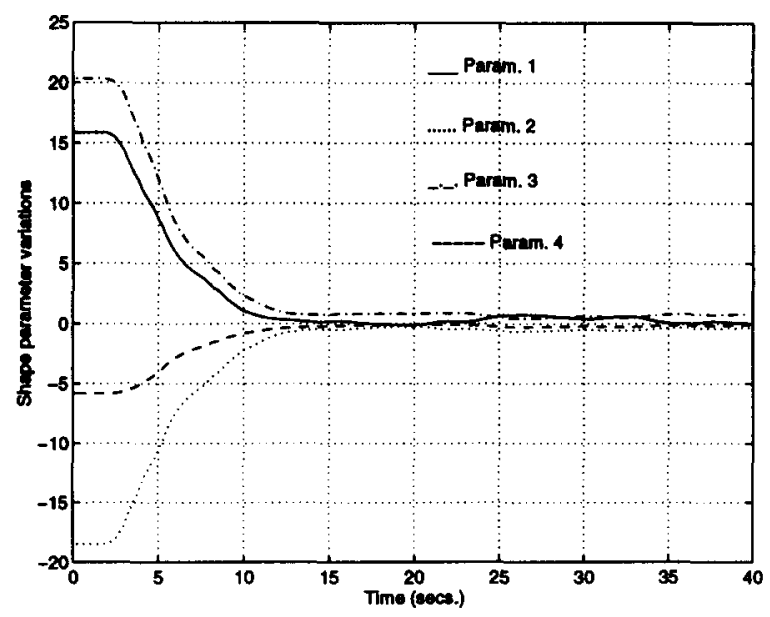

Fig. 9. Parametric shape-profile variations (nominal)

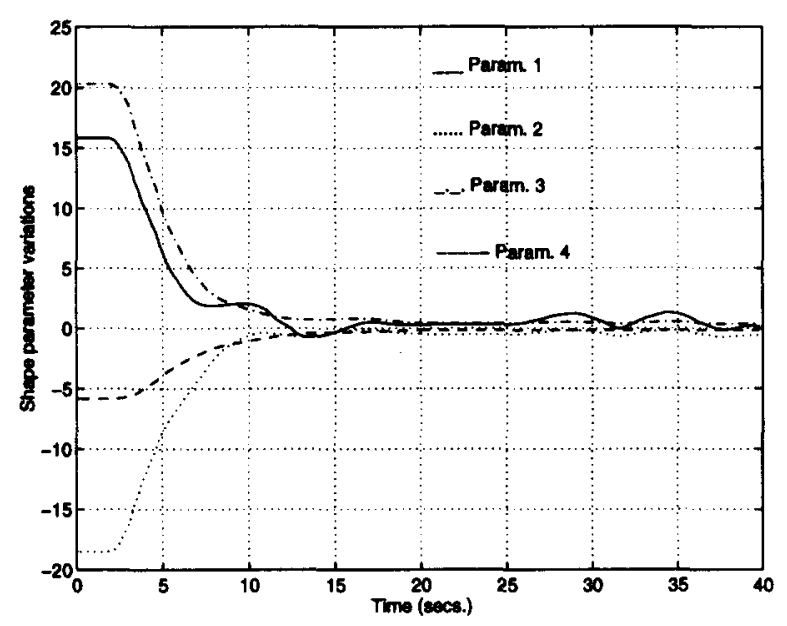

Fig. 10. Parametric shape-profile variations (Pass 1)

\section{DISCUSSION OF APPROACH}

Several comments regarding the approach taken, together with the outline results achieved, are pertinent. Firstly, the $\mathrm{H}_{\infty}$ control-design methodology provides an analytical methodology for controller determination, with a guarantee of robust stability across a given set of plant perturbations. Some user input is required in terms of weight function selection, in order to specify the desired controller properties, but this specification will be consistent across different plants. Such a solution procedure is in contrast to previous approaches, which have tended to involve a considerable amount of user input (e.g., for frequency response shaping), and retention of stability across different passes must be checked separately for each individual case. One of the principal benefits, therefore, in adopting the $\mathrm{H}_{\infty}$ methodology is the provision of an automated design philosophy, which can provide a systematic means of developing a set of controllers to cover all passes and schedules.

The dimension reduction, which was employed in Section 3.2, accords with other design approaches, but is clearly motivated by the inability to formulate a design for the full $8 \times 8$ system which has reasonable sensitivity properties.

Some further benefits of the $\mathrm{H}_{\infty}$ design, which are not explicitly stated in the specification, are also available. In particular, it is known that the mill matrices produced from the static models of Gunawardene (1982) and Dutton (1983) contain modelling inaccuracies. The $\mathrm{H}_{\infty}$ controller naturally produces some immunity to such errors, by providing good stability margins and insensitivity to d.c. parameter variations. These benefits extend to unmodelled (soft) nonlinear dynamics (Safonov, 1980), which are known to be present in the actuators. Further nonlinear effects may manifest themselves in the real system, since the mill matrices produced by Gunawardene's model are linearised gain matrices.

A final comment concerns controller implementation. Observation of the controller state-space description highlights the possible need to employ controller order-reduction techniques. This will be the subject of further investigation.

\section{CONCLUSIONS}

$\mathrm{H}_{\infty}$ has been shown to be a sound framework in which the Sendzimir mill shape control problem may be tackled. The synthesis of a controller which actively builds in robustness to known parameter variations is particularly significant when the multi-pass, multischedule nature of the mill operation is considered. The design philosophy can also form an important building block upon which to base a systematic solution to the controller scheduling problem.

\section{REFERENCES}

Dutton, K. (1983) An investigation into the design and performance of a shape control system for a Sendzimir mill, PhD Dissertation, Sheffield City Polytechnic.

Gunawardene, G.W.D.M. (1982) Static model development for the Sendzimir cold rolling mill, PhD Dissertation, Sheffield City Polytechnic.

Jaeckel, K. (1993) Private communication. 
Maciejowski, J.M. (1989) Multivariable Feedback Design, Addison Wesley.

Ringwood, J.V., D.H. Owens and M.J. Grimble (1990) Feedback design of a canonical multivariable system with application to shape control in Sendzimir mills, Trans. ASME, Journal of Dynamic Syst., Meas. and Control, 116, 104-110.

Ringwood, J.V. (1994) Diagonalisation of a class of multivariable system via an actuator linearisation technique, Proc. IMC-11, Belfast, N.Ireland.

Ringwood, J.V. (1995) Multivariable control using the singular value decomposition in steel rolling with quantitative robustness assessment, Control Engineering Practice, 3, 495-503.

Safonov, M.G. (1980) Stability and robustness of multivariable feedback systems, MIT Press, Camb., Mass.

Safonov, M.G. and R.Y.Chiang (1988) Robust Control Toolbox for Use With MATLAB®.

$\mathrm{H}_{\infty}$ controller state-space description (Jordan form):

$$
\operatorname{eig}(A)=\left[\begin{array}{l}
-1.6076+2.6923 j \\
-1.6076-2.6923 j \\
-3.5069 \\
-6.4409 \\
-5.8667 \\
-4.8711 \\
-3.0574+5.3484 j \\
-3.0574-5.3484 j \\
-2.2788+3.9461 j \\
-2.2788-3.9461 j \\
-2.7722+4.4383 j \\
-2.7722-4.4383 j \\
-0.0100 \\
-0.0100 \\
-0.0100 \\
-0.0100
\end{array}\right]
$$

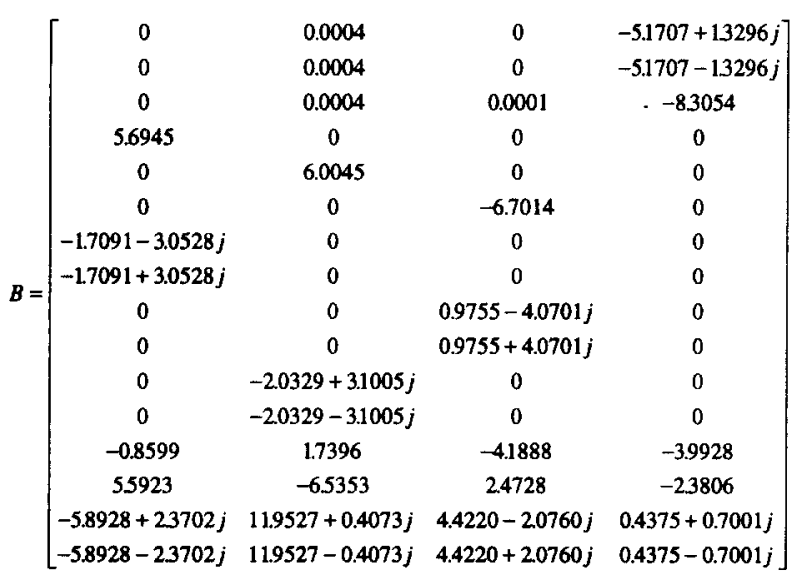

\section{APPENDIX}

$\mathrm{G}_{\mathrm{a}}$ for Pass 1 of schedule:

$G_{1}=\left[\begin{array}{ccccccccc}5.09092 & 6.34360 & 0.26968 & -2.48203 & -2.80510 & -2.38248 & -1.98539 & -2.02650 \\ 1.00279 & 3.34942 & 3.13050 & 0.25597 & -2.05200 & -2.61747 & -2.22675 & -2.24159 \\ -0.89666 & 0.48619 & 3.37291 & 2.50732 & -0.51331 & -2.36358 & -2.43742 & -2.42733 \\ -1.34573 & -1.48967 & 1.38566 & 3.30285 & 1.94524 & -0.89787 & -2.33347 & -2.34295 \\ -1.17256 & -2.25602 & -0.98889 & 1.75450 & 3.35399 & 1.52936 & -1.48323 & -1.56488 \\ -1.00443 & -2.38581 & -2.32983 & -0.62499 & 2.31819 & 3.36104 & 0.72382 & 0.63545 \\ -0.94654 & -2.29830 & -2.69351 & -2.21713 & -0.16058 & 2.83896 & 3.68777 & 3.78202 \\ -0.85555 & -1.87405 & -2.08204 & -2.37710 & -1.96711 & 0.59551 & 5.93841 & 6.06127\end{array}\right]$

$G_{\mathrm{a}}$ for Pass 6 of schedule:

$G_{6}=\left[\begin{array}{cccccccc}4.12077 & 4.50166 & -0.19453 & -2.07818 & -2.13894 & -1.88670 & -1.72470 & -1.69745 \\ 0.48345 & 2.34648 & 2.35850 & 0.18784 & -1.37801 & -1.73299 & -1.54856 & -1.52501 \\ -0.72171 & 0.45367 & 2.35850 & 0.18784 & -1.37801 & -1.73299 & -1.54856 & -1.53501 \\ -0.94952 & -0.96559 & 1.07289 & 2.50512 & 1.41015 & -0.63816 & -1.57128 & -1.53163 \\ -0.82447 & -1.52496 & -0.62953 & 1.40829 & 2.51223 & 1.09019 & -1.04530 & -1.00397 \\ -0.71384 & -1.58239 & -1.49821 & -0.26633 & 1.87309 & 2.51739 & 0.38262 & 0.41848 \\ -0.69047 & -1.54612 & -1.73372 & -1.38238 & 0.10433 & 2.20707 & 2.36437 & 2.38232 \\ -0.79992 & -1.75596 & -1.86316 & -2.15344 & -1.87756 & 0.04788 & 4.66098 & 4.45294\end{array}\right]$

$$
D=\left[\begin{array}{llll}
0 & 0 & 0 & 0 \\
0 & 0 & 0 & 0 \\
0 & 0 & 0 & 0 \\
0 & 0 & 0 & 0
\end{array}\right]
$$

$C^{I}=\left[\begin{array}{cccc}0 & 0 & 0 & -0.3703+0.6589 j \\ 0 & 0 & 0 & -0.3703-0.6589 j \\ 0 & 0 & 0 & -0.5416 \\ 1.1543 & 0 & 0 & 0 \\ 0 & 1.0485 & 0 & 0 \\ 0 & 0 & -0.8501 & 0 \\ -1.3274+0.1567 j & 0 & 0 & 0 \\ -1.3274-0.1567 j & 0 & 0 & 0 \\ 0 & 0 & -0.7583-0.7282 j & 0 \\ 0 & 0 & -0.7583+0.7282 j & 0 \\ 0 & 0.6538+1.0507 j & 0 & 0 \\ 0 & 0.6538-10507 j & 0 & 0 \\ -0.0003 & 0.0008 & -0.0041 & -0.0306 \\ 0.0029 & 0.0009 & 0.0060 & -0.0213 \\ 0.0007+0.0036 j & 0.0015+0.0029 j & 0.0020-0.0028 j & -0.0039+0.0098 j \\ 0.0007-0.0036 j & 0.0015-0.0029 j & 0.0020+0.0028 j & -0.0039-0.0098 j\end{array}\right]$

